\title{
Análise comparativa do desempenho acústico de sistemas de fachada com esquadrias de PVC com persiana e diferentes tipos de vidros em ensaios de laboratório
}

\author{
Comparative analysis of the acoustic performance of \\ facades with PVC frames with shutters and different \\ types of glass in laboratory tests
}

\section{Cristiane Cassol Schvarstzhaupt Bernardo Fonseca Tutikian Maria Fernanda de Oliveira Nunes}

\section{Resumo}

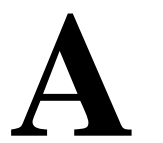

s janelas proporcionam iluminação e ventilação no interior das edificações e exercem uma grande influência no conforto acústico dentro dos ambientes, assim como em demais elementos constituintes do sistema de fachada. No sul e sudeste do Brasil, é comum o uso de persianas junto às esquadrias para proporcionar sombreamento, o que pode ser positivo para o desempenho acústico. Muitas vezes se especifica vidros de espessuras mais elevadas para aumentar o desempenho acústico do sistema de fachada, sem, no entanto, entender a real contribuição deste elemento no desempenho global. Este artigo, portanto, tem a finalidade de apresentar os resultados obtidos em ensaios de laboratório de esquadrias de PVC de dois modelos, utilizando vidros simples de $4 \mathrm{~mm}$ e $6 \mathrm{~mm}$, laminados com vidros $4+4 \mathrm{~mm}$ e 4+6mm e vidro duplo de 4+6mm, com câmara de ar entre estes de $6 \mathrm{~mm}$ de espessura, nas situações de persiana aberta e fechada. Os ensaios foram realizados de acordo com a norma ISO 10140-2 (INTERNATIONAL..., 2010), referenciada na norma brasileira NBR 15575-4 (ABNT, 2013). Os resultados obtidos mostram que a isolação sonora de sistema de fachada é superior na situação de esquadria

Cristiane Cassol Schvarstzhaupt Programa de Pós-Graduação em Engenharia Civil Universidade do Vale do Rio dos Sinos Av. Unisinos, 950, Cristo Rei. São Leopoldo - RS - Brasil CEP 90510-030 Tel.: (51) 3591-1122

E-mail: cris.cassol@gmail.com

Bernardo Fonseca Tutikian itt Performance Universidade do Vale do Rio dos Sinos E-mail: bftutikian@unisinos.br

Maria Fernanda de Oliveira Nunes Laboratório de Tecnologia Construtiva Universidade de Caxias do Sul Av. Frederico Segala, 3099, Samuara Caxias do Sul - RS - Brasil CEP 95010-550

Tel.: (54) 3289-9000 Ramal 9031 E-mail: mfonunes@ucs.br

Recebido em 09/03/14 Aceito em 20/10/14 com persiana fechada, exceto nas baixas frequências. $\mathrm{O}$ vidro laminado apresentou melhores resultados em relação ao vidro duplo, porém com pequena diferença em relação ao sistema com vidros simples.

Palavras-chaves: Desempenho acústico. Janelas. Persiana. Vidros.

\section{Abstract}

Windows offer daylight and fresh air in buildings and play an important role in ensuring acoustic comfort indoors. In Southern and Southeastern Brazil, rolling shutters are commonly used in windows to provide shading, and they can also have a positive effect on acoustic performance. Often, higher-thickness glass is indicated in order to increase the acoustic performance of the facade system, without, however, understanding the real contribution of this elementto the overall performance. This study presents the results obtained from laboratory tests with two models of PVC frame windows. The glass used was monolithic glass, with $4 m m$ and $6 m m$ thickness, laminated glass with 4+4mm and 4+6mm thickness, and double glazed glass with $4+6 \mathrm{~mm}$, with a $6 \mathrm{~mm}$ chamber, in situations with extended and retracted rolling shutters. The measurements were applied according to the ISO 10140-2 (INTERNATIONAL..., 2010) standard referred to the Brazilian standard NBR 15575-4 (ABNT, 2013). The results obtained show that the window sound insulation is higher when the rolling shutter is extended, except on low frequencies. Laminated glass presented better results compared with monolithic glass and double glazed glass.

Keywords: Acoustic performance. Sound insulation. Rolling shutter. PVC frame window. 


\section{Introdução}

Devido à alta incidência da luz solar sobre as fachadas é comum o uso de persianas junto às esquadrias. Esses elementos são constituídos por réguas que cobrem em guias e formam um painel de enrolar, que se aloja em uma caixa na parte superior da janela. As persianas permitem o sombreamento das aberturas e, ao mesmo tempo, a troca de ar entre o espaço interno e o externo. No entanto, a eficiência acústica desses elementos pode ser questionada e, diante das atuais alterações normativas, o desempenho acústico das janelas com persianas produzidas no Brasil deve ser analisado.

Dependendo da fonte sonora, os ruídos exteriores podem ser transmitidos para o interior do edifício através de dois meios principais, de acordo com o comprimento de onda. Sons de alta frequência, percebidos pelo ouvido humano como sons agudos, com menor comprimento de onda, propagam-se nos fechamentos através de frestas, orifícios e outras aberturas pelas quais o ar pode penetrar. Os sons com maior comprimento de onda, de baixa frequência, percebidos como graves, apresentam o fenômeno da difração em sua propagação e tendem a contornar obstáculos e incidir em fechamentos externos, o que ocasiona a transmissão para o interior dos edifícios através de vibrações (HASSAN, 2009).

Os fechamentos externos usualmente são compostos de mais de um elemento construtivo, e a inserção de uma janela numa fachada acarreta perdas no desempenho acústico dos fechamentos de alvenaria, que poderiam apresentar desempenho acústico satisfatório. Por isso, o desempenho acústico da janela deve ser semelhante ao da parede, para que não ocorra perda na eficiência no isolamento sonoro global do fechamento (MOONEY, 2012). No entanto, o quanto uma janela pode comprometer o desempenho acústico de um fechamento depende dos índices de redução sonora do fechamento e da janela isoladamente, das áreas ocupadas de cada elemento e da forma como o caixilho é instalado (JONES, 2008).

Algumas questões práticas devem ser analisadas para qualificar o desempenho acústico de uma janela e, considerando-se os diferentes componentes utilizados em sua fabricação, pode-se indicar os principais como o vidro (tipo $\mathrm{e}$ espessura), o tipo de abertura da janela (garantia de estanqueidade, sistema de fechamento) e o material do caixilho (HASSAN, 2009; MOONEY, 2012).

O comportamento acústico de um painel, vidro simples, apresenta variações de acordo com a frequência do som incidente. Pode-se classificar esse comportamento em diferentes regiões por faixa de frequência. Em baixas frequências, antes de ocorrerem as principais ressonâncias do painel da partição, a rigidez desempenha um papel importante no aumento do isolamento acústico. $\mathrm{Na}$ região de transição entre baixas e médias frequências, ocorrem as ressonâncias do painel, para o qual o amortecimento reduzirá a amplitude da vibração de ressonância e, portanto, aumentará o isolamento. Nas médias frequências a massa do painel é o fator mais importante, e o acréscimo da massa da partição melhora o isolamento sonoro, com o aumento de $6 \mathrm{~dB}$ para cada duplicação da massa. Em frequências mais elevadas, outro tipo de ressonância ocorre, decorrente de ondas de flexão na partição. O efeito dessas ondas no índice de redução de ruído é chamado de efeito coincidência, com a queda no isolamento acústico (região de coincidência), as quais começam acima da frequência crítica. Os efeitos do amortecimento são importantes nessa região, para limitar as amplitudes de vibração de ressonância e minimizar a queda do isolamento pela coincidência. Nas baixas frequências, região da ressonância, varia conforme Peters (2013).

O comportamento acústico dos vidros varia conforme sua espessura e composição. Os sistemas constituídos por mais de um painel de vidro apresentam resultados superiores de isolamento acústico. Os vidros duplos utilizam um sistema massa-mola-massa, cujo amortecimento da vibração é dado pelo ar. O som incidente em um elemento duplo é transmitido através da primeira superfície para o sistema de amortecimento, que reduz a transmissão sonora para a outra superfície (HARRIS, 1997). O vidro laminado, composto de painéis de vidro unidos por uma película de polivinilbutiral (PVB), pode ser fabricado em diferentes espessuras de película, mas maior espessura do PVB não assegura a melhora no desempenho acústico da janela (HASSAN, 2009). Tanto o vidro duplo quanto o laminado apresentam desempenho acústico superior ao do vidro comum, por proporcionarem um acoplamento elástico entre os painéis de vidro.

O elemento que faz a vedação do vidro com o caixilho apresenta significativa influência no isolamento acústico de janelas de vidro monolítico, devido a melhorias das condições de borda. Nesse sentido, o uso de material resiliente como elemento de vedação eleva a capacidade de amortecimento do sistema e, consequentemente, seu desempenho acústico. Logo, nas janelas com folhas móveis o isolamento acústico proporcionado pelo vidro pode

136 Schvarstzhaupt, C. C.; Tutikian, B.; Nunes, M. F. de O. 
não ser significativo se não houver garantia de estanqueidade de todo o sistema de fechamento (UTLEY; FLETCHER, 1969). Nesse caso, o isolamento acústico nas altas e médias frequências fica comprometido (VINOKUR, 1991).

Díaz e Pedrero (2009) realizaram ensaios em laboratório com esquadrias de PVC de duas folhas, no sistema de correr, com diferentes espessuras de vidros duplos, nas situações de persiana aberta e fechada. Segundo os autores, a persiana fechada atua como um painel que, juntamente com o vidro duplo, funciona como um sistema de painéis triplos. O índice de redução sonora ponderado $(R w)$ da janela, nas altas frequências, é consideravelmente superior quando a persiana está fechada em relação a quando está aberta. Porém, nas frequências nas quais os modos normais de vibração estão situados, há diminuição no $R w$, com pior desempenho nessa situação.

Os autores apresentaram, ainda, ensaios com a inserção de diferentes materiais na caixa de persiana,com o objetivo de verificar alterações no desempenho acústico em relação à caixa vazia. Foram utilizados dois materiais, poliestireno expandido e fibra mineral, para reduzir o volume de ar no interior da caixa. Os resultados mostram melhora no Rnas altas frequências, mas não houve alteração nos valores globais. Dessa forma, a colocação de material no interior das caixas de persiana não foi decisiva para melhorar o $R w$ das janelas nesse estudo. Por outro lado, Díaz e Pedrero (2009) afirmam que um fator importante para o aumento do desempenho acústico da janela é o fechamento eficiente da tampa da caixa de persiana.

López, Escudero e Rozas(2010) realizaram ensaios com janelas de PVC de abrir e oscilobatente, com persianas com caixa integrada, guias de alumínio com uma ou mais folhas móveis e vidros duplos de diversas composições. Além das medições com as persianas abertas e fechadas, os pesquisadores estudaram a influência do revestimento da caixa da persiana com material de absorção sonora e a vedação da abertura para saída da persiana. A colocação de material de absorção no interior da caixa da persiana também não apresentou influência significativa nos resultados, mas a vedação da abertura de saída da folha da persiana proporcionou aumentos entre 3 e $4 \mathrm{~dB}$ no $R w$ das janelas com caixilhos de abrir.

Outras pesquisas também apresentam resultados semelhantes aos citados anteriormente, em ensaios em laboratório e em campo, com vidros simples e duplos, também nas situações de persiana aberta e fechada.Segundo esses estudos, os valores globais de isolamento sonoro são superiores na situação de persiana fechada. Porém, nas baixas frequências, região controlada pela ressonância, o isolamento acústico é menor na situação de persiana fechada que na de persiana aberta. Isso ocorre devido ao volume que se forma entre a persiana e o vidro, que resulta em um sistema acústico ressonante, aumentando a energia sonora em certos modos de vibração (DÍAZ; DÍAZ; NAVACERRADA, 2013; PATRÍCIO; BRAGANÇA, 2004).

Nas fachadas com janelas de vidro simples, quando a persiana está fechada, o isolamento sonoro da fachada, $R w$, melhora em média $2,5 \mathrm{~dB}$ quando comparado à situação de persiana aberta. A melhora é reduzida para $1,5 \mathrm{~dB}$ quando a janela é de vidro duplo (DÍAZ; DÍAZ; NAVACERRADA, 2013).

Apesar da existência de diversos estudos que indicam o desempenho acústico de esquadrias com persianas, cabe ressaltar que essas pesquisas foram desenvolvidas fora do Brasil, com materiais e sistemas diferentes dos nacionais. As diferenças mais relevantes, que evidenciam a importância de estudos no Brasil, estão na composição do fechamento opaco do sistema de vedação vertical e nos materiais que compõem os perfis dos caixilhos. Além disso, pode-se citar também as diferenças nos processos construtivos, como, por exemplo, de execução da argamassa de assentamento (FERREIRA NETO; BERTOLI, 2010).

A norma brasileira que estabelece o desempenho de sistemas de vedações verticais é a NBR 15575 4 (ABNT, 2013), que apresenta os requisitos, critérios e métodos para a avaliação de desempenho. O requisito referente ao desempenho acústico indica o critério de isolação acústica ao ruído aéreo, e, de acordo com o texto, os ensaios em campo têm o objetivo de verificar a isolação sonora de um sistema de vedação em edificações concluídas. Já os ensaios em laboratório têm a finalidade de determinar a isolação sonora de elementos construtivos, como janelas, o que fornece valores de referência de cálculo para projetos.

No Quadro 1 são apresentados os níveis de desempenho mínimo, intermediário e superior da referida norma, conforme o índice de redução sonora ponderado, $R w$, para componentes de fachada em ensaios em laboratório. Além dos índices de redução sonora, também é considerado o ruído urbano, conforme as classes de ruído, que são estipuladas subjetivamente.

Segundo os critérios da Norma, o mesmo sistema com determinado valor de $R w$ pode apresentar classificações distintas de desempenho, conforme o local de implantação do edifício. Para fins dessa 
classificação, o maior condicionante é o ruído originado de meios de transporte,o que pode condicionar os sistemas de vedação.

Diante do exposto, o objetivo deste trabalho é analisar o desempenho acústico de janelas com persianas, considerando-se as variáveis mecanismo de fechamento, tipo e espessura de vidro, acionamento de persiana através de ensaios em laboratório, e classificação de desempenho segundo a NBR 15575-4 (ABNT, 2013).

\section{Método}

O método de ensaio adotado para determinar em laboratório o isolamento sonoro do sistema de fechamento segue as recomendações da NBR 15575-4 (ABNT, 2013), que remete aos procedimentos da ISO 10140-2 (INTERNATIONAL..., 2010).

Os ensaios foram realizados em câmara reverberante no Instituto Tecnológico em Desempenho da Construção Civil (itt Performance), na Unisinos, em cooperação interinstitucional com o Laboratório de Tecnologia Construtiva (LBTEC), da UCS.

\section{Determinação do índice de redução sonora}

No interior da câmara foi construída uma parede de blocos cerâmicos que divide o espaço em câmara de emissão e câmara de recepção, conforme ilustrado nas Figuras 1 e 2, e as esquadrias foram instaladas conforme as especificações dos fabricantes. $\mathrm{O}$ volume da câmara de emissão é de $57,60 \mathrm{~m}^{3}$, e da câmara de recepção, de $51,60 \mathrm{~m}^{3}$, com área total da parede de $14 \mathrm{~m}^{2}$.

O índice de redução sonora, $R$, foi determinado através de ensaios em bandas de um terço de oitava de 100 a $5.000 \mathrm{~Hz}$. Os equipamentos utilizados da Bruel\&Kjaer foram: fonte sonora dodecaédrica 4296; amplificador de potência 2716; calibrador acústico 4231; e analisador sonoro 2270 .

Quadro 1 - Níveis de desempenho para componentes de fachada para ensaios em laboratório

\begin{tabular}{|c|c|c|c|}
\hline $\begin{array}{l}\text { Classe } \\
\text { de ruído }\end{array}$ & Localização da habitação & $\begin{array}{l}\text { Rw } \\
(\mathbf{d B})\end{array}$ & $\begin{array}{c}\text { Nível de } \\
\text { desempenho }\end{array}$ \\
\hline \multirow{3}{*}{ I } & \multirow{3}{*}{$\begin{array}{l}\text { Habitação localizada distante de fontes de ruído intenso de } \\
\text { quaisquer naturezas. }\end{array}$} & $\geq 25$ & Mínimo \\
\hline & & $\geq 30$ & Intermediário \\
\hline & & $\geq 35$ & Superior \\
\hline \multirow{3}{*}{ II } & \multirow{3}{*}{$\begin{array}{l}\text { Habitação localizada em áreas sujeitas a situações de ruído } \\
\text { não enquadráveis nas classes I e III. }\end{array}$} & $\geq 30$ & Mínimo \\
\hline & & $\geq 35$ & Intermediário \\
\hline & & $\geq 40$ & Superior \\
\hline \multirow{3}{*}{ III } & \multirow{3}{*}{$\begin{array}{c}\text { Habitação sujeita a ruído intenso de meios de transporte e } \\
\text { de outras naturezas, desde que esteja de acordo com a } \\
\text { legislação. }\end{array}$} & $\geq 35$ & Mínimo \\
\hline & & $\geq 40$ & Intermediário \\
\hline & & $\geq 45$ & Superior \\
\hline
\end{tabular}

Fonte: NBR 15575-4 (ABNT, 2013).

Figura 1 - Planta baixa esquemática da câmara de reverberaçãodividida em sala de emissão e sala de recepção

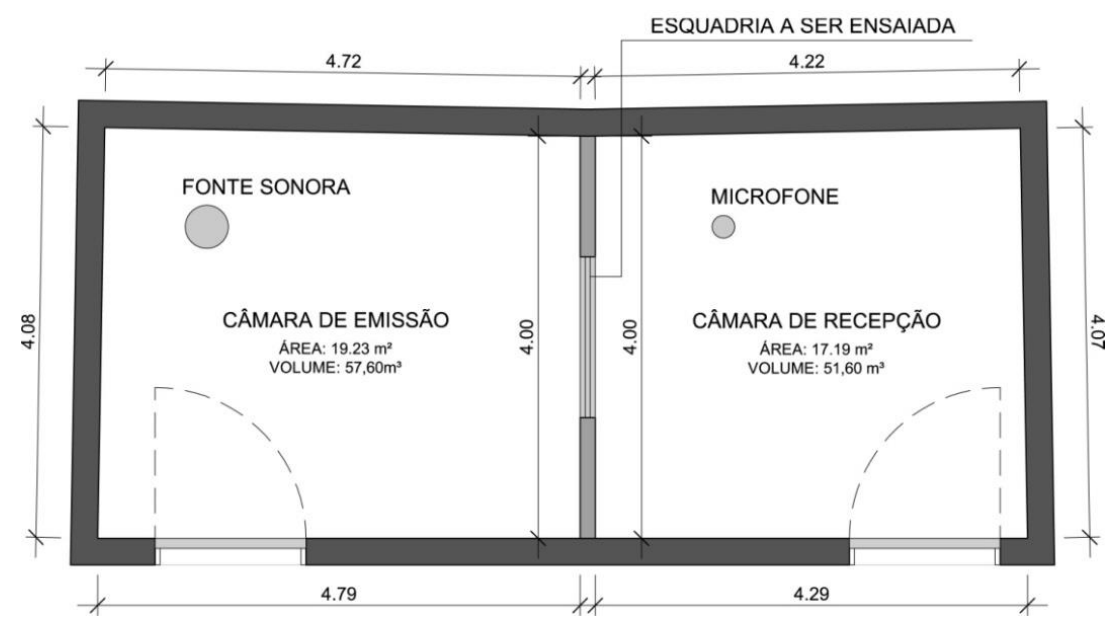

138 Schvarstzhaupt, C. C.; Tutikian, B.; Nunes, M. F. de O. 
Figura 2 - Câmara de ensaio com a esquadria instalada

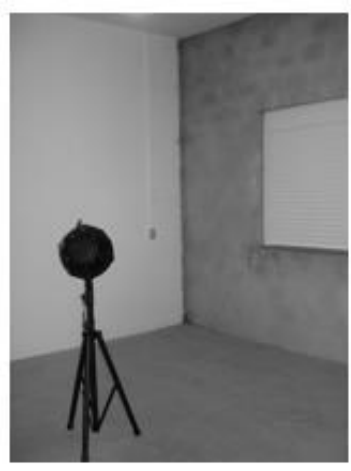

(a)

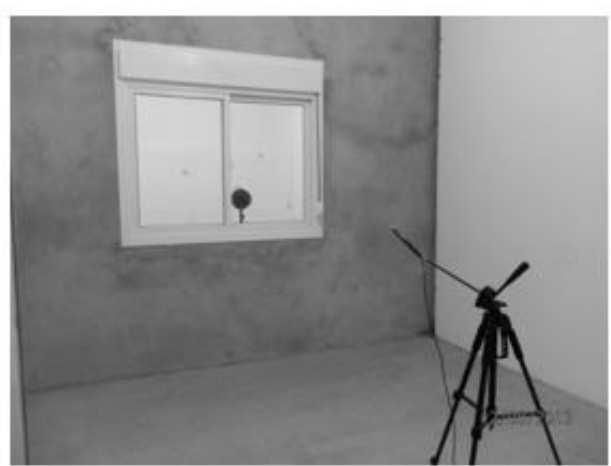

(b)
Na câmara (Figura 2) de emissão é medido o nível de pressão sonora da fonte, $L 1$, e na câmara de recepção são medidos o nível de pressão sonora, $L 2$, o ruído de fundo e o tempo de reverberação, $T$, necessários para obter o índice de redução sonora, $R$, dado na Equação 1:

$R=L 1-L 2+10 \log \left(\frac{S}{A}\right)$

Eq. 1

Onde:

Ll é o nível médio de pressão sonora na câmara de emissão, em dB;

L2 é o nível médio de pressão sonora na câmara de recepção, em dB;

$S$ é a área do elemento em ensaio, $\mathrm{em} \mathrm{m}^{2}$; e

$A$ é a área de absorção sonora equivalente na câmara de recepção, em m².

O valor de $R$ é obtido para cada frequência. Para tornar possível a classificação do elemento analisado em função do isolamento de ruído aéreo, o valor que pode ser apresentado através de um único número para expressar os resultados obtidos para cada banda de frequência é o Índice de Redução Sonora Ponderado, $R w$. Este valor é obtido através da comparação do espectro de $R$ com a curva de referência padrão indicada na norma ISO 717-1 (INTERNATIONAL..., 2013).

\section{Esquadrias ensaiadas}

Foram realizados ensaios em sistemas de vedação vertical externa com dois modelos de esquadrias compostas de perfis de PVC rígido, estrutura interna em aço galvanizado, vedações entre vidro $\mathrm{e}$ perfil em borracha EPDM, guias dos caixilhos em alumínio e deságue através de câmaras independentes dos reforços.

O modelo A (Figura 3) possui fechamento através de maçaneta com cremona na folha interna esquerda e fecho oculto na folha externa direita, enquanto o modelo B (Figura 4) possui fecho central do tipo caracol. Os dois modelos também se diferenciam pelo tipo de perfil: o modelo A, com perfil mais robusto, suporta vidros de até 22 $\mathrm{mm}$, enquanto o modelo B suporta vidros de até 6 mm. Ambos possuem dimensões de $1,60 \mathrm{~m}$ de largura por $1,40 \mathrm{~m}$ de altura, duas folhas de correr e caixa de persiana embutida.

No modelo A foram ensaiados os tipos de vidros apresentados no Quadro 2.

No modelo B foi ensaiado o vidro monolítico comum, liso, transparente, de espessura $6 \mathrm{~mm}$, espessura máxima suportada por esse modelo.

Os ensaios foram realizados com persianas abertas e fechadas. 
Figura 3 - Modelo de esquadria A com cremona na folha interna esquerdae fecho oculto na folha externa direita
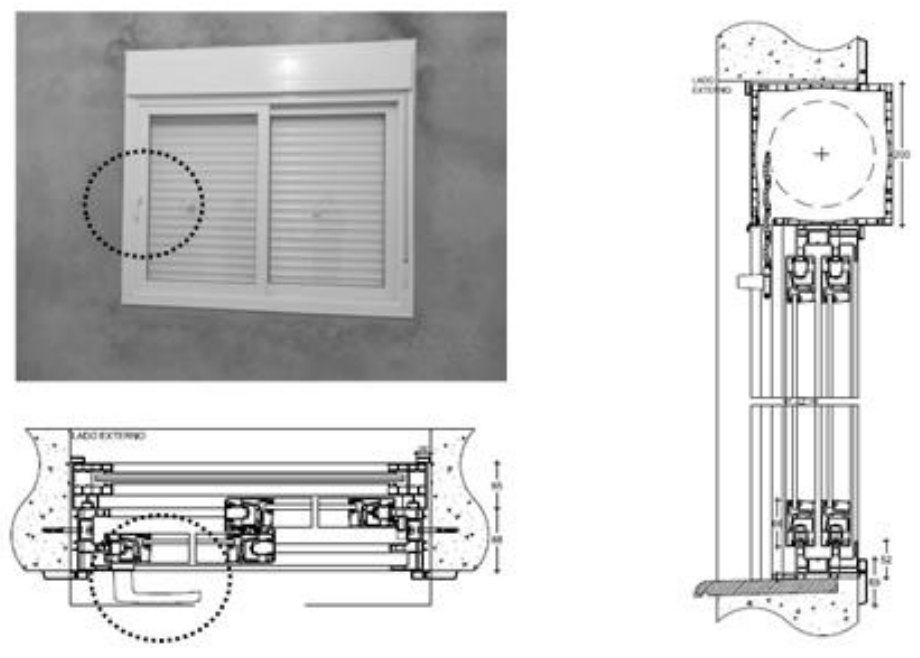

Figura 4 - Modelo de esquadria B com fecho central do tipo caracol
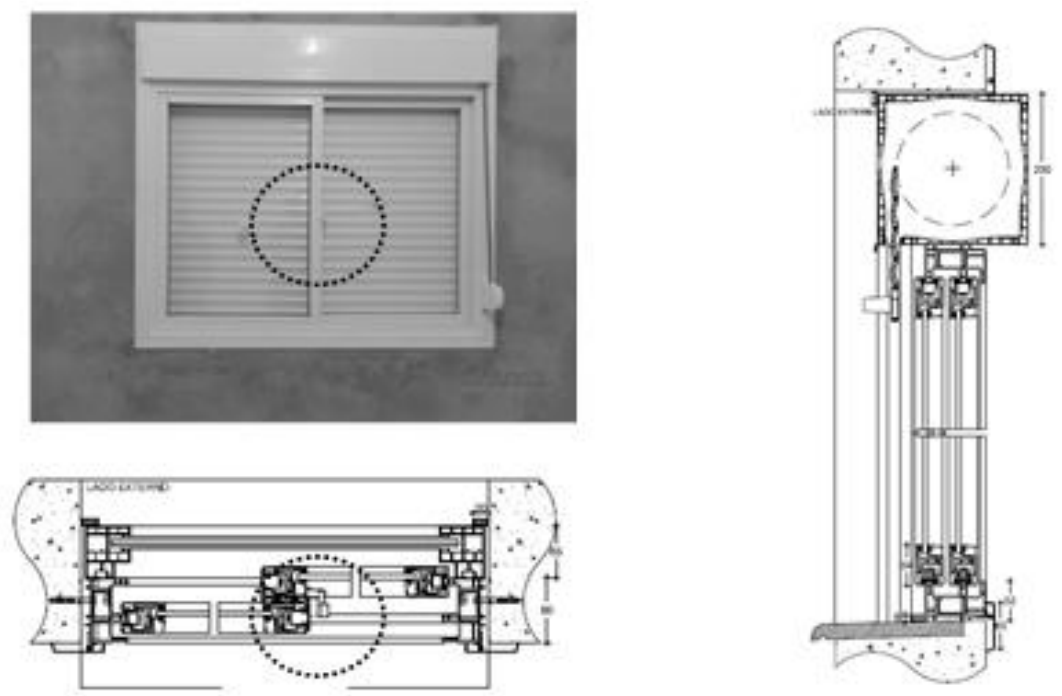

Quadro 2 - Especificação dos vidros e nomenclatura adotada no trabalho

\begin{tabular}{|c|c|c|}
\hline Tipo de vidro & Espessuras (mm) & Nomenclatura adotada \\
\hline \multirow{2}{*}{ Monolítico comum, liso e transparente } & 4 & C 4 \\
\cline { 2 - 3 } & 6 & C 6 \\
\hline $\begin{array}{c}\text { Laminado liso, transparente, constituído de dois } \\
\text { vidros comuns e um filme de PVB }\end{array}$ & $4+4$ & L 4+4 \\
\cline { 2 - 3 } & $4+6$ & L 4+6 \\
\hline Duplo com dois vidros comuns e câmara de ar & $4(6) 6^{*}$ & D 4(6)6
\end{tabular}

Nota: *O valor entre parênteses representa a espessura da câmara de ar em $\mathrm{mm}$. 


\section{Resultados}

\section{Influência do mecanismo de fechamento}

A Figura 5 apresenta o gráfico com os valores de Rem função da frequência obtidos nos ensaios com os modelos de esquadria A e B, com a persiana aberta e fechada, todos com o vidro de $6 \mathrm{~mm}$.

Comparando-se os dois modelos de esquadrias ensaiadas com vidro de $6 \mathrm{~mm}$, os valores obtidos de $R$ são maiores na situação de persiana fechada, com aumento da isolação nas altas frequências. Comparando-se os modelos A e B, verifica-se que, com a persiana fechada, os valores são semelhantes nas frequências de 500 a $2.000 \mathrm{~Hz}$, e, para as demais frequências, as diferenças não são consideradas significativas. Com a persiana aberta, os perfis gráficos para os dois modelos são semelhantes para a maior parte do espectro analisado, e as maiores diferenças foram verificadas nas frequências de 250 e $5.000 \mathrm{~Hz}$. Portanto, observa-se que aumentar as dimensões dos perfis e alterar o mecanismo de fechamento da esquadria, para a espessura de $6 \mathrm{~mm}$, não influi nos resultados de desempenho acústico de persiana fechada, para todas as frequências analisadas.

Devido à semelhança entre os resultados dos dois modelos e da possibilidade de maior variação na composição dos vidros, a apresentação dos resultados em gráficos nos itens seguintes considera apenas o modelo de esquadria A.

\section{Influência do tipo de vidro com a persiana aberta}

A Figura 6 apresenta o gráfico com os valores de $R$ em função da frequência obtidos nos ensaios do sistema de vedação vertical externa com a esquadria modelo A com a persiana aberta e distintos tipos e espessuras de vidros.

Os resultados obtidos nos ensaios com persiana aberta mostraram que, de modo geral, a alteração do tipo e espessura dos vidros pouco influiu no resultado final. Porém, o modelo A com vidro laminado de $4+6 \mathrm{~mm}$ possui $R$ maior que nos demais vidros analisados. Os vidros comuns de 4 $\mathrm{mm}$ e $6 \mathrm{~mm}$ apresentaram os menores valores de $R$, o que era esperado em função da reduzida espessura dos vidros simples; e os vidros laminado 4+4 $\mathrm{mm}$ e duplo apresentaram valores intermediários, com melhor isolamento do vidro duplo entre 200 e $800 \mathrm{~Hz}$ e do vidro laminado 4+4 $\mathrm{mm}$ nas frequências acima de $3.150 \mathrm{~Hz}$. Observase, ainda, que os perfis gráficos das composições de vidros analisadas indicam elevação na capacidade de isolamento nas frequências até 200 $\mathrm{Hz}$, menor variação nos valores nas frequências entre 200 e $630 \mathrm{~Hz}$, e aumento nos valores até $5.000 \mathrm{~Hz}$, que apresenta os maiores valores.

\section{Influência do tipo de vidro com a persiana fechada}

Na Figura 7 pode-se observar comparativamente os valores de $R$ em função da frequência obtidos nos ensaios do sistema de vedação vertical externa com a esquadria modelo A com a persiana fechada e distintos tipos e espessuras de vidros.

Figura 5 - Índice de redução sonora, $R$, dos modelos A e B com vidro simples de $6 \mathrm{~mm}$ nas situações de persiana aberta (PA) e persiana fechada (PF)

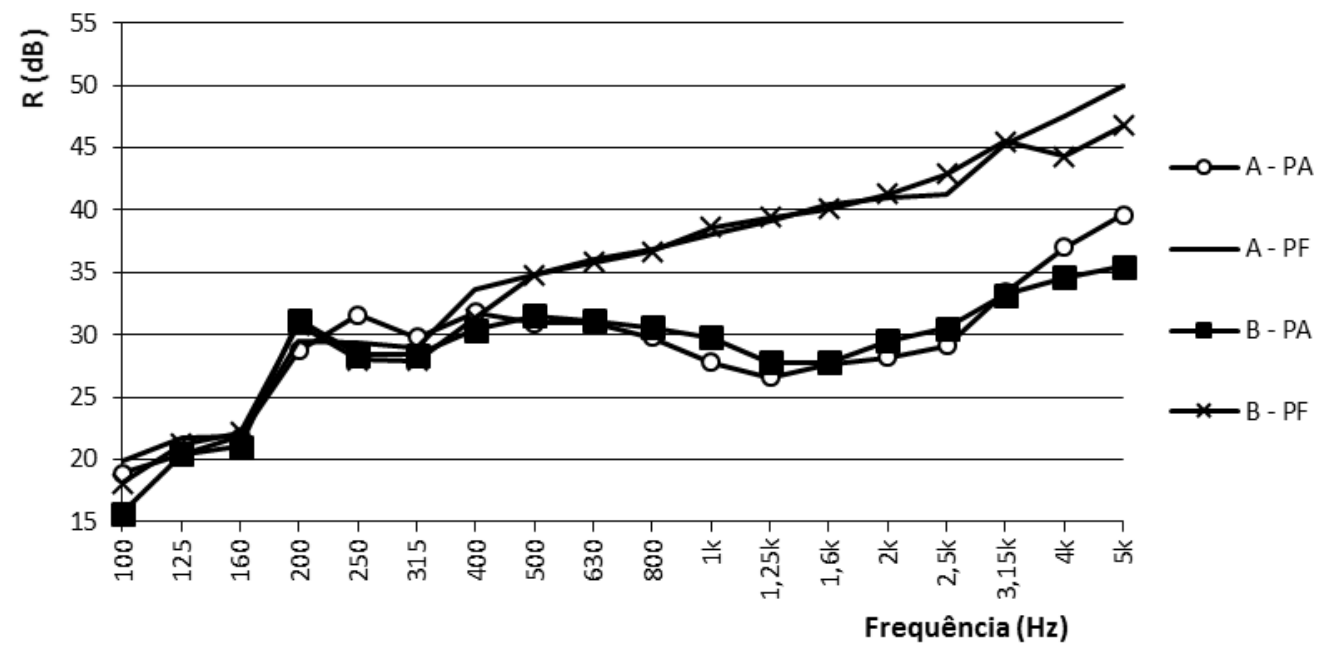


Figura 6 - Índice de redução sonora, $R$, no modelo de esquadria A com vidros comum, laminado e duplo nas espessuras de $4 \mathrm{~mm}$ e $6 \mathrm{~mm}$, na situação de persiana aberta

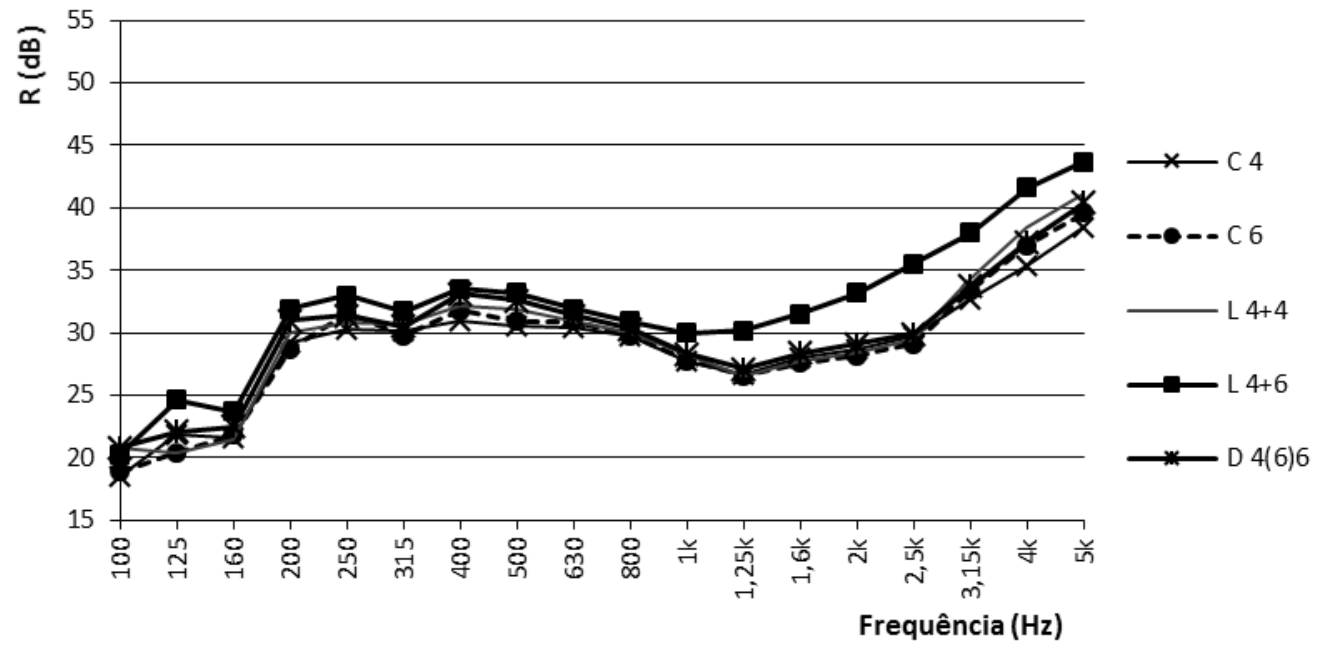

Figura 7 - Índice de redução sonora, R, no modelo de esquadria A com vidros comum, laminado e duplo nas espessuras de $4 \mathrm{~mm}$ e $6 \mathrm{~mm}$, na situação de persiana fechada

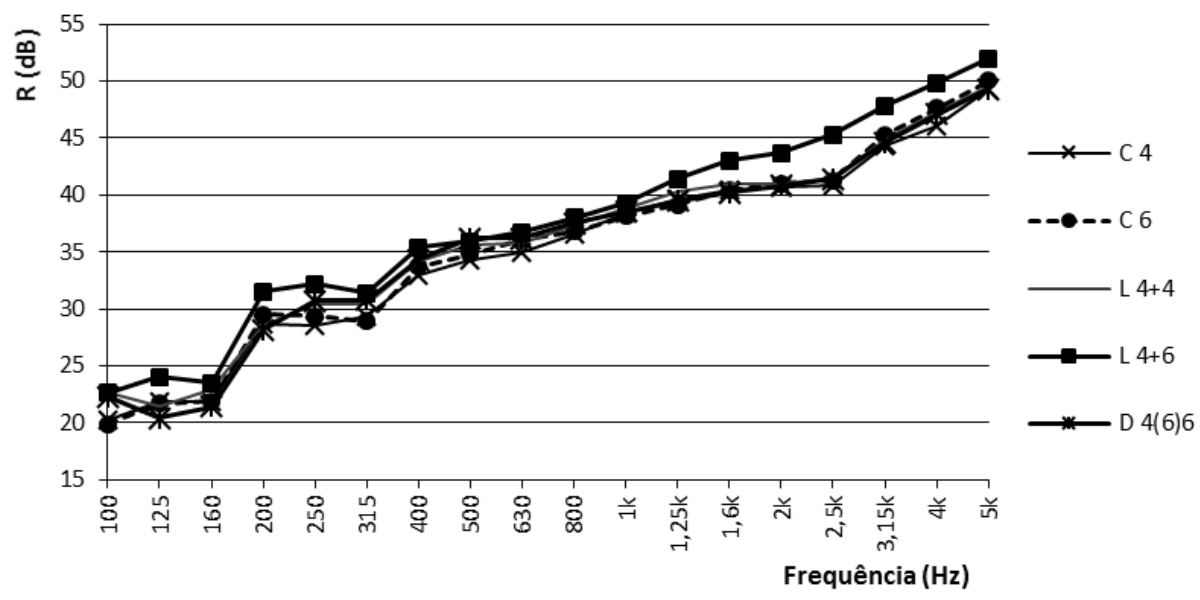

Os resultados obtidos nos ensaios com a persiana fechada mostram que a alteração do tipo e da espessura de vidro pouco influiu no resultado final, como já ocorrera com a persiana aberta. Entretanto, ainda é possível observar que, na maior parte do espectro, o $R$ é superior na esquadria de modelo A com vidro laminado $4+6 \mathrm{~mm}$. Nas frequências entre 125 e $250 \mathrm{~Hz}$, o vidro duplo de $4+6+6 \mathrm{~mm}$ foi o que apresentou menores valores de $R$. O comportamento de $R$ nas esquadrias com a persiana fechada mostra um comportamento de aumento da isolação com o aumento da frequência, destacando-se o decaimento a $315 \mathrm{~Hz}$, identificado com frequência de ressonância da alvenaria, e a 2,5 $\mathrm{kHz}$ a frequência crítica dos vidros, que é anulada no vidro laminado $4+6 \mathrm{~mm}$.

\section{Comparação entre os vidros laminados e duplo}

A Figura 8 apresenta o gráfico com os valores de $\mathrm{R}$ em função da frequência obtidos nos ensaios do sistema de vedação vertical externa com esquadrias modelo A com vidros laminados e duplos, para situações de persiana aberta e fechada.

Os resultados obtidos nos ensaios com a esquadria de modelo A com vidro laminado $4+6 \mathrm{~mm}$ foram os que apresentam melhores valores de $R$ quando comparados aos demais vidros, tanto na situação de persiana aberta quanto fechada. Nas frequências acima de $400 \mathrm{~Hz}$, os ensaios realizados com a persiana fechada obtiveram valores superiores de $R$ em relação aos resultados obtidos com a persiana aberta. Já nas frequências abaixo de $400 \mathrm{~Hz}$ os valores nas duas situações são bastante próximos, inclusive com valores de $R$ superiores em 
algumasfrequências na situação aberta em relação à situação fechada.

\section{Índices de redução sonoro ponderados}

O Quadro 3 mostra os resultados do índice de redução sonoro ponderado para os sistemas verticais de vedação externa com os dois modelos de esquadria, diferentes tipos e espessuras de vidros e com a persiana aberta e fechada, com as respectivas classificações de desempenho segundo classes de ruído da NBR 15575-4 (ABNT, 2013).

Os valores de $R w$ são superiores na situação de persiana fechada em relação à de persiana aberta em todas as situações. A diferença chega a $9 \mathrm{~dB}$ de uma situação para outra no modelo de esquadria
A,nos vidros simples de $6 \mathrm{~mm}$, laminado de $4+4$ mm e duplo 4(6)6 mm. O modelo de esquadria A com vidro laminado $4+6 \mathrm{~mm}$ foi o que apresentou maior valor de $R w$ em ambas as situações, de persiana aberta e fechada.

As janelas com vidro comum e persianas abertas atendem ao desempenho mínimo somente se a habitação estiver localizada distante de fontes de ruído intenso, e nas demais situações em que existe proximidade com fontes de ruído não atenderia à classificação mínima. Com as persianas fechadas, todos os sistemas analisados alcançaram níveis de desempenho previstos na NBR 15575-4 (ABNT, 2013), no entanto a classificação é reduzida à medida que o local de implantação for considerado mais ruidoso.

Figura 8 - Índice de redução sonora, $R$, da esquadria de modelo A com vidros laminados e duplonas situações de persiana aberta (PA) e persiana fechada (PF)

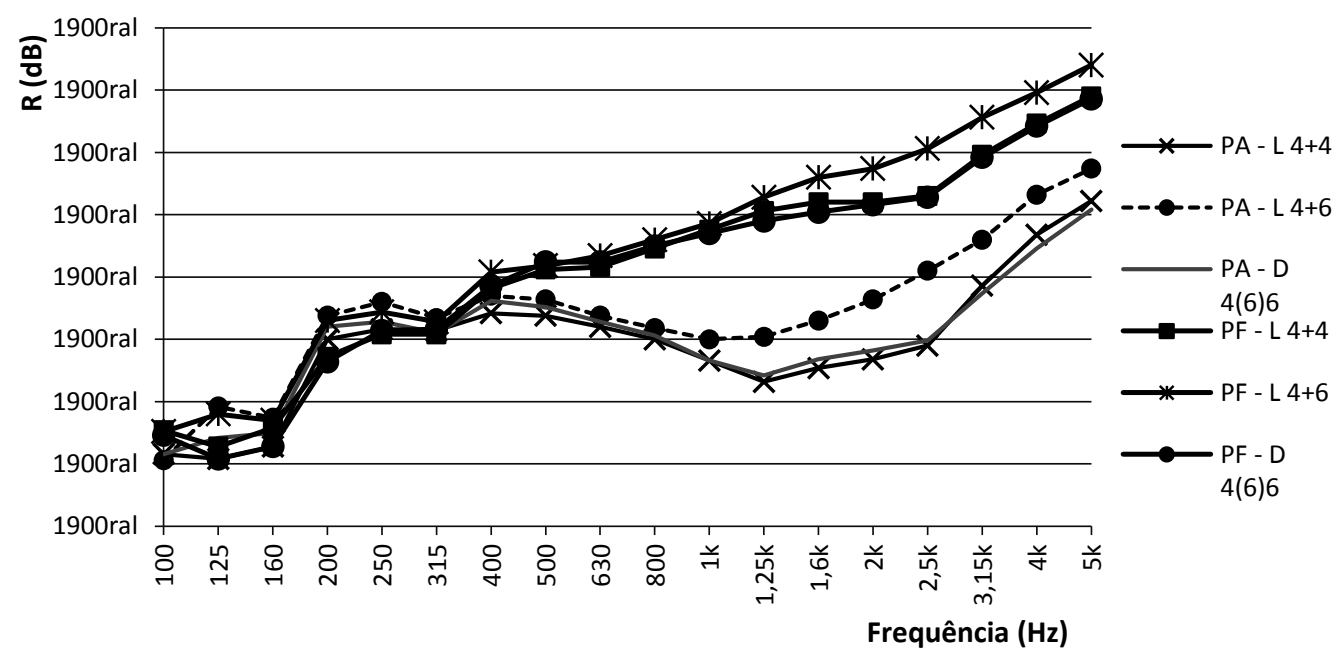

Quadro 3 - Resultados em $R w$ e as respectivas classificações de desempenho

\begin{tabular}{|c|c|c|c|c|c|c|c|c|c|}
\hline \multirow{3}{*}{ Esquadria } & \multirow{3}{*}{ Tipo de vidro } & \multicolumn{2}{|c|}{ Rw (dB) } & \multicolumn{6}{|c|}{ Desempenho conformea classe de ruído } \\
\hline & & \multirow{2}{*}{ PA } & \multirow{2}{*}{$\mathbf{P F}$} & \multicolumn{2}{|c|}{$\mathbf{I}$} & \multicolumn{2}{|c|}{ II } & \multicolumn{2}{|c|}{ III } \\
\hline & & & & PA & PF & $\mathbf{P A}$ & PF & $\mathbf{P A}$ & PF \\
\hline \multirow{5}{*}{ Modelo A } & $\mathrm{C} 4$ & 29 & 37 & $\mathrm{M}$ & $\mathrm{S}$ & N/A & $\mathrm{I}$ & N/A & $\mathrm{M}$ \\
\hline & $\mathrm{C} 6$ & 29 & 38 & $\mathrm{M}$ & $S$ & N/A & $\mathrm{I}$ & N/A & $\mathrm{M}$ \\
\hline & $\mathrm{L} 4+4$ & 30 & 38 & I & $\mathrm{S}$ & $\mathrm{M}$ & I & N/A & $\mathrm{M}$ \\
\hline & $\mathrm{L} 4+6$ & 33 & 39 & $\mathrm{I}$ & $S$ & $M$ & $\mathrm{I}$ & N/A & $\mathrm{M}$ \\
\hline & D 4(6)6 & 30 & 38 & $\mathrm{I}$ & $\mathrm{S}$ & $\mathrm{M}$ & $\mathrm{I}$ & N/A & $\mathrm{M}$ \\
\hline Modelo B & C 6 & 30 & 37 & I & $\mathrm{S}$ & $\mathrm{M}$ & I & N/A & $\mathrm{M}$ \\
\hline
\end{tabular}

Nota: Legenda:
M - desempenho mínimo;
I - desempenho intermediário;
$\mathrm{S}$ - desempenho superior;
N/A - não atende;
PA - persiana aberta; e
PF - persiana fechada. 


\section{Conclusões}

Os dados de desempenho acústico de sistemas de fechamentos assumem importância crescente diante da entrada em vigor da norma NBR 15575-4 (ABNT, 2013).

Observou-se neste trabalho que o desempenho acústico do vidro isoladamente não constitui uma informação tão significativa para o sistema, em função do comportamento do conjunto dos componentes: placa da persiana, sistema de vedação na caixa e mecanismo de fechamento das folhas.

O desempenho das esquadrias na situação de persiana fechada é superior em relação à situação de persiana aberta, quando considerados os valores de $R w$. Isso ocorre devido à contribuição do painel externo formado pela persiana, o que resulta em um sistema duplo ou triplo. No entanto, essa diferença não é a mesma nos diferentes tipos de vidro. Nos vidros comuns, verificaram-se as maiores diferenças, de 8 e $9 \mathrm{~dB}$, enquanto nos sistemas com vidro laminado $4+6 \mathrm{~mm} \quad 0$ fechamento da persiana apresentou uma contribuição de $6 \mathrm{~dB}$ no $R w$. Dessa forma, pode-se afirmar que o uso da persiana fechada em sistemas com vidros simples é fundamental para a qualificação do isolamento acústico do conjunto.

$\mathrm{O}$ vidro laminado de espessura $4+6 \mathrm{~mm}$ foi o que apresentou maiores valores de $R$, maiores inclusive que vidro duplo 4(6)6mm. Quando comparados os valores de $R w$, o vidro laminado alcançou $3 \mathrm{~dB}$ a mais na situação de persiana aberta, e $1 \mathrm{~dB}$ na situação de persiana fechada, quando comparado ao vidro duplo.

A pequena diferença de desempenho entre os modelos de esquadrias A e B com o vidro de $6 \mathrm{~mm}$ provavelmente ocorreu devido à ligação do vidro com o caixilho, tendo em vista que a diferença nos valores é percebida em frequências controladas pelo amortecimento.

A classe de ruído urbano é um fator decisivo na classificação de desempenho acústico de sistemas de vedações verticais externas, e a atual subjetividade de enquadramento pode resultar em interpretações equivocadas.

\section{Referências}

\section{ASSOCIAÇÃO BRASILEIRA DE NORMAS TÉCNICAS. NBR 15575-4: edifícios:}

habitacionais: desempenho: parte 4: requisitos para os sistemas de vedaçoes verticais internas e externas - SVVIE. Rio de Janeiro, 2013.
DÍAZ, C.; DÍAZ, A.; NAVACERRADA, M. A. An Experimental Study on the Effect of Rolling Shutters on the Field Measurements of Airborne Sound Insulation of Façades. Applied Acoustics, v. 74, n. 1, p. 134-140, jan. 2013

DÍAZ, C.; PEDRERO, A. An Experimental study on the Effect of Rolling Shutters and Shutter Boxes on the Airborne Sound Insulation of Windows. Applied Acoustics, v. 70, n. 2, p. 369 377, fev. 2009.

FERREIRA NETO, M. F.; BERTOLI, S. R. Desempenho Acústico de Paredes de Blocos e Tijolos Cerâmicos: uma comparação entre Brasil e Protugal. Ambiente Construído, Porto Alegre, v. 9, n. 2, p. 87-103, abr./jun. 2010.

HARRIS, D. Noise Control Manual For

Residential Buildings. New Jersey: McGraw-Hill Education, 1997.

HASSAN, O. A. B. Building Acoustics and Vibration: theory and practice. London: World Scientific Publishing Company, 2009.

INTERNATIONAL ORGANIZATION FOR STANDARDIZATION. ISO 717-1: acoustics: rating of sound insulation in buildings and of buildings elements: part 1: airborne sound insulation. Geneva, 2013.

INTERNATIONAL ORGANIZATION FOR STANDARDIZATION. ISO 10140-2: acoustics: laboratory measurement of sound insulation of building elements: part 2: measurement of airborne sound insulation. Geneva, 2010.

JONES, D. Acoustical Noise Control. In: Handbook For Sound Engineers. Oxford: Taylor \& Francis, 2008.

LÓPEZ, J.; ESCUDERO, S.; ROZAS, M. J. de. Ventana y Cajón de Persiana: estudio del comportamiento acústico en diferentes situaciones. In: CONGRESO NACIONAL DE ACÚSTICA $6^{\mathrm{O}}$ CONGRESO IBÉRICO DE ACÚSTICA, 41., León, 2010Anais... León: Sociedad Española de Acústica, 2010.

MOONEY, J. W. Practical Architectural Acoustics Notebook. New York: KJWW, 2012.

PATRÍCIO, J.; BRAGANÇA, L. The Contribution of Roller Shutters to Noise Insulation of Façades.

Building Acoustics, v. 11, n. 4, p. 309-324, 1 dez. 2004.

PETERS, R. J. Acoustics and Noise Control. New Jersey: Taylor \& Francis, 2013.

UTLEY, W. A.; FLETCHER, B. L. Influence of Edge Conditions on the Sound Insulation of Windows. Applied Acoustics, v. 2, n. 2, p. 131136, apr. 1969. 
VINOKUR, R. Y. The Influence of linear sound bridges linking panel edges on the airborne sound insulation of double partitions. Applied Acoustics, v. 34 , p. $19-35,1991$.

\section{Cristiane Cassol Schvarstzhaupt}

Programa de Pós-Graduação em Engenharia Civil | Universidade do Vale do Rio dos Sinos | Av. Unisinos, 950, Cristo Rei. São Leopoldo RS - Brasil | CEP 90510-030 | Tel.: (51) 3591-1122 | E-mail: cris.cassol@gmail.com

\section{Bernardo Fonseca Tutikian}

itt Performance | Universidade do Vale do Rio dos Sinos | E-mail: bftutikian@unisinos.br

\section{Maria Fernanda de Oliveira Nunes}

Laboratório de Tecnologia Construtiva | Universidade de Caxias do Sul | Av. Frederico Segala, 3099, Samuara | Caxias do Sul - RS - Brasil | CEP 95010-550 | Tel.: (54) 3289-9000 Ramal 9031 | E-mail: mfonunes@ucs.br

\section{Revista Ambiente Construído}

Associação Nacional de Tecnologia do Ambiente Construído

Av. Osvaldo Aranha, $99-3^{\circ}$ andar, Centro

Porto Alegre - RS - Brasil

$$
\text { CEP } 90035-190
$$

Telefone: +55 (51) 3308-4084

Fax: +55 (51) 3308-4054

www.seer.ufrgs.br/ambienteconstruido

E-mail: ambienteconstruido@ufrgs.br 